\title{
Review
}

\section{Interventions to optimize antimicrobial stewardship}

\author{
Nick J. Tinker PharmD ${ }^{1}$ (1), Rachel A. Foster PharmD, MBA ${ }^{1}$, Brandon J. Webb MD², Souha Haydoura MD², \\ Whitney R. Buckel PharmD ${ }^{3}$ and Edward A. Stenehjem MD, MSc ${ }^{2}$ \\ ${ }^{1}$ Pharmacy Services, Intermountain Medical Center, Murray, Utah, ${ }^{2}$ Division of Infectious Diseases and Clinical Epidemiology, Intermountain Healthcare, Murray, \\ Utah and ${ }^{3}$ Pharmacy Services, Intermountain Healthcare, Murray, Utah
}

\begin{abstract}
Developing and improving an antimicrobial stewardship program successfully requires evaluation of numerous factors. As technology progresses and our understanding of antimicrobial resistance grows, careful consideration should be taken to ensure that a program meets the needs of the institution and is achievable given the available resources. In this review, we explore fundamental initiatives and strategies for both new and established antimicrobial stewardship programs, including the specific areas to target and key elements required for sustainable implementation.
\end{abstract}

(Received 1 September 2021; accepted 27 September 2021)

Multiple strategies can be deployed by an antimicrobial stewardship program (ASP) to improve antimicrobial prescribing in the acute-care setting. How an ASP selects and implements these interventions depends on many factors. Each ASP is unique and should be tailored to function in its specific healthcare environment.

In this review, we highlight multiple antimicrobial stewardship interventions shown to improve antimicrobial prescribing and the care of hospitalized patients. Rather than implementing all interventions immediately, the selection of ASP interventions should be based on an understanding of the hospital needs and the goals of the ASP. Performing a needs assessment is an efficient method of identifying areas on which an ASP should focus with new or expanded interventions. The Center for Disease Control and Prevention (CDC) Core Elements of Hospital Antibiotic Stewardship Programs Checklist is a needs assessment that provides a framework for identifying hospital ASP opportunities. ${ }^{1}$

When selecting antimicrobial stewardship interventions, we recommend focusing on 3 key principles. First, adapt and tailor antimicrobial stewardship interventions to the resources available. Such resources include the ASP team's available time, availability of infectious diseases (ID) consultation, involvement of ID clinicians in ASP activities, the capabilities of the electronic health record (EHR), and hospital culture. Second, implement antimicrobial stewardship interventions across the continuum of inpatient care. Opportunities to improve antimicrobial prescribing occur throughout a patient's admission, arising at different

Author for correspondence: Nick J. Tinker, Intermountain Medical Center, Infectious Diseases Clinic, 5171 S Cottonwood St, Building 1 - Suite 350, Murray, UT 84107. E-mail: ntinker86@gmail.com

Cite this article: Tinker NJ, et al. (2021). Interventions to optimize antimicrobial stewardship. Antimicrobial Stewardship of Healthcare Epidemiology, https://doi.org/ 10.1017/ash.2021.210 points. Third, engage frontline clinicians when selecting and implementing new antimicrobial stewardship interventions. When developing any new intervention, it is paramount to understand the attitudes, motivations, and intentions of those whose behavior is targeted for change. ${ }^{3}$ Engaging frontline clinicians in antimicrobial stewardship discussions and decisions will build rapport and demonstrate your commitment to patient care.

\section{Fundamental interventions}

ASPs should select and implement interventions that will target high-priority opportunities and goals. ASPs should prioritize antimicrobial stewardship interventions that have demonstrated improved prescribing and patient care in the medical literature. We have labeled these evidence-based antimicrobial stewardship interventions as fundamental interventions. Fundamental interventions are backed by the strongest clinical evidence and are considered the foundation of an ASP. ${ }^{4}$ Fundamental interventions are adaptable to all hospitals regardless of size and stewardship personnel, and they target opportunities to improve prescribing across the continuum of care by engaging with frontline clinicians to build interdisciplinary relationships.

\section{Facility-specific treatment guidelines}

The establishment of facility-specific treatment guidelines for common ID syndromes is fundamental to optimizing empiric antimicrobial selection, de-escalation, and duration of therapy. Facility-specific treatment guidelines can be developed from national guidelines and modified based on local microbiology susceptibility data, formulary options, and available resources. Lower respiratory tract infections, urinary tract infections (UTIs), and skin and soft-tissue infections (SSTIs) account for most

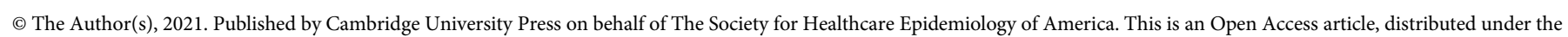

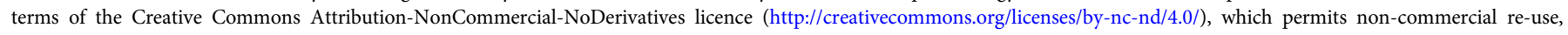

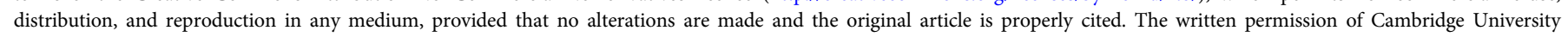
Press must be obtained prior to any commercial use and/or adaptation of the article. 
antibiotic use in hospitals ${ }^{5}$ and should be a high priority for hospital-based ASPs.

Facility-specific treatment guidelines are best developed collaboratively in multidisciplinary teams. Involving clinical stakeholders will increase the likelihood that recommendations are accepted. These guidelines can be used by the ASP when performing postprescription and prior authorization reviews and can be used as an audit tool when evaluating appropriate antimicrobial use retrospectively.

Integration of these guidelines into the clinician's workflow can be achieved by leveraging the EHR to incorporate recommendations into order sets and clinical decision support systems (CDSSs). Current and clinically useful guidelines that have been developed with key stakeholders will improve the likelihood of clinician acceptance and integration.

\section{Prospective audit and feedback}

A prospective audit and feedback (PAF) program allows the ASP to review patient encounters and identify opportunities where management can be optimized. PAF reviews are often initiated based on the antimicrobial prescribed or by clinical results obtained from the microbiology laboratory.

\section{Postprescription review}

Postprescription review (PPR) involves a member of the ASP who performs a comprehensive patient review after an antimicrobial is prescribed to optimize treatment. This review can result in recommendations to change the agent, to adjust the dose or duration of therapy, to convert intravenous to oral formulations, and to evaluate drug-drug interactions. When deployed effectively, PPR can reduce overall antibiotic use, cost, and length of stay (LOS). This review can be performed immediately after prescribing or within 24-72 hours after prescribing when more clinical information is available. Incorporating PPR into an ASP requires consideration of the hospital's needs and resources, and prioritizing specific agents or patients to review is vital.

Aligning the PPR targets with the goals of the ASP ensures that the reviewer's time is used optimally. The specific approach to implementing PPR can and should look different at each hospital; it should account for the unique requirements and characteristics of the facility.

In addition to ASP personnel, other resources contribute to the success of PPR; some are arguably essential for success in large hospitals. Prominent examples of crucial resources needed include information technology (IT) and access to specially trained antimicrobial stewardship personnel. Leveraging technology to aid in PPR can reduce the total number of patients requiring review and the time spent per patient. 6,7 Many modern EHRs and CDSSs can identify patients on specific antimicrobials for review by the ASP. Physicians and pharmacists trained in infectious diseases (ID) are ideally suited to perform PPR and to provide recommendations. Hospitals without access to ID-trained specialists can utilize interested physicians and pharmacists to perform PPR. Appropriately trained staff can review more patients with greater complexity and can provide more appropriate recommendations. ${ }^{8}$ Formal training with certification can be obtained online through various institutions, including the Society for Infectious Diseases Pharmacists, Making a Difference in Infectious Diseases, and the Society for Healthcare Epidemiology of America.
Microbiology and laboratory surveillance-focused prospective audit and feedback

PAF can also be initiated from clinical results obtained from the microbiology or clinical laboratory. Just like PPR, laboratoryinitiated interventions should be prioritized based on the critical nature of the test result and institution-specific needs discussed previously. ASPs with limited personnel or resources may focus on the review of sterile site cultures that pose a high risk of morbidity and mortality if treated inappropriately. In contrast, well-staffed ASPs may review all positive cultures to evaluate for de-escalation opportunities. Other examples include positive multidrug-resistant pathogens, positive cultures in patients not on antibiotic therapy, and infection-related biomarkers, serologies, or viral studies. Notably, positive Clostridioides difficile tests have been shown to be a high-yield stewardship intervention. ${ }^{9}$

Molecular rapid diagnostic tests (mRDTs) have emerged as a key technology to decrease the time to identification and antimicrobial susceptibility of pathogens. These tests can decrease the time to effective therapy and antimicrobial optimization, and they also decrease LOS in patients with bloodstream infections. The clinical impact of mRDTs is most pronounced when reviewed and acted upon by the ASP in real time. ${ }^{10,11}$ Successful implementation of mRDTs is dependent on workflows supporting antimicrobial stewardship review of mRDTs results and on communicating recommendations to frontline clinicians. Incorporating mRDTs results into the ASP workflow should be a high priority in hospitals that utilize this technology.

\section{Prior authorization}

Another fundamental practice of antimicrobial stewardship is prior authorization, sometimes called antimicrobial restriction. Prior authorization requires certain agents to be approved prior to initiation. This typically requires the prescriber to contact a member of the ASP for authorization, typically based on policies jointly established by the facility's ASP and the pharmacy and therapeutics committee. Common reasons for restriction include drug cost, complexity in dosing and monitoring, and broadspectrum agents that should only be used to treat specific pathogens.

Prior authorization allows direct control over specific agents and has been shown to decrease designated antimicrobial use, to decrease cost, and to improve antibiotic resistance among gramnegative pathogens. ${ }^{12} \mathrm{~A}$ mature prior authorization program can manage difficulties with acquisition and shortages by restricting drugs as needed. Instituting a prior authorization program requires the ASP to consider many operational and logistic challenges. Ideally, members with ID training will review the requests, although establishing approval criteria for restricted agents can allow non-ID trained clinicians to participate and ensure consistency of approvals. ${ }^{13}$ Since prompt antimicrobial therapy is extremely important in critically ill patients, approval logistics need to be addressed before starting a prior authorization program, particularly in facilities that cannot provide around-the-clock reviews. ASPs with heavy prior authorization requirements can have disadvantages as well. The perceived impedance to provider autonomy has been shown to create a negative perception of antimicrobial stewardship and to damage the rapport necessary for effective collaboration ${ }^{14}$; it also threatens provider efficiency. ${ }^{15}$ Actively managing these concerns and ensuring prior authorization requests provide collaborative and educational opportunities to instill a team-based approach to improving patient care. 


\section{Postprescription review versus prior authorization}

Postprescription review and prior authorization have long been identified as the 2 pillars of antimicrobial stewardship interventions. Results of direct comparisons have been published and demonstrate that PPR results in greater improvements in durations of therapy and antibiotic appropriateness compared to prior authorization, as well as more collaboration between the antimicrobial stewardship team and prescribers. ${ }^{16,17}$ Despite this difference, many experts suggest that these interventions are synergistic, and both should remain priorities for implementation. A hospitals can use local data and predefined goals to determine how much of each intervention to blend into its ASP.

\section{Expanded practices}

In contrast to the fundamental practices, expanded practices have not yet become standard antimicrobial stewardship practices for all hospitals. Many of the expanded practices described here have a significant body of evidence supporting their practice. However, incorporating them into an ASP is often limited by the resources and specialized knowledge required. Although establishing the fundamental practices of antimicrobial stewardship should be prioritized, several expanded practices can be evaluated and implemented concurrently based on need and resources available.

\section{Diagnostic stewardship}

Rapid and accurate diagnosis of infection is critical for appropriate antimicrobial initiation and subsequent optimization. However, when used inappropriately, diagnostic tests can identify organisms that represent colonization or contamination, leading to an incorrect diagnosis. Common targets include inappropriate urine cultures or C. difficile tests, where positive results may lead to treatment with unnecessary antibiotics and may delay the identification of the true cause of their symptoms. ${ }^{18}$ Inappropriate testing can also result in overdiagnosis of healthcare-associated infections, increasing a hospital's publicly reported infection rate with subsequent downstream consequences. ${ }^{19}$

Unnecessarily testing patients without infectious symptoms or with a low pre-test probability of infection increases the likelihood of a false-positive test result. Diagnostic stewardship aims to improve the appropriate use and interpretation of diagnostic tests to guide therapeutic decisions and avoid the consequences of falsepositive results, while also reducing costs. Diagnostic stewardship interventions are becoming increasingly common among hospitals, and many of the interventions are under the direction of ASPs.

\section{Discharge antimicrobial stewardship: Oral antibiotic therapy at the time of discharge}

Multiple studies have demonstrated that for common infections (eg, UTI, community-acquired pneumonia, and SSTI), approximately two-thirds of the total antibiotic treatment course is completed after the patient is discharged, often with broadspectrum, suboptimal agents. ${ }^{20,21}$ Studies of pneumonia have shown that $57 \%$ of patients have antibiotics prescribed at discharge, accounting for $39 \%$ of the overall duration and $93 \%$ of total excess duration, most often with fluoroquinolones. ${ }^{22-24}$ Additionally, $44 \%$ of discharge antibiotics for UTIs are due to treatment of asymptomatic bacteriuria. ${ }^{24}$ Antimicrobial stewardship interventions focusing solely on inpatient use will affect only a fraction of the total use, thus coordinated discharge antimicrobial stewardship interventions are needed. Interventions can include a timeout checklist, medication reconciliation by trained pharmacists, provider education, EHR alerts, and incorporating discharge recommendations during stewardship led PPR.

\section{Discharge antimicrobial stewardship: Outpatient parenteral antibiotic therapy (OPAT) review}

OPAT utilization has increased over the past 20 years, allowing more patients on IV antimicrobials to be discharged and to complete their course of therapy outside the hospital. OPAT has been shown to improve patient satisfaction and to reduce hospital LOS, healthcare costs, and the potential of healthcare-associated infections. ${ }^{25}$ Patients receiving OPAT are inherently complex and result in hospital readmission rates exceeding those of nonOPAT patients, and an estimated $15 \%-30 \%$ of OPAT discharges are for patients eligible for oral therapy. ${ }^{26,27}$ Safe OPAT requires (1) a thorough review of appropriateness, (2) close follow-up with the prescribing clinician, (3) appropriate lab monitoring, and (4) an established duration. Effective OPAT programs are supported by infrastructure that allows for consistent review of labs and communication between the agency providing the antimicrobials, treating clinicians, and the patient.

The role of the ASP in OPAT is evolving. In some institutions, the ID consult service manages all OPAT via required ID consultation. This procedure ensures that patients are appropriately evaluated prior to discharge and that follow-up is established. Not all hospitals have the resources to support mandatory ID consultation for OPAT, and ASPs may facilitate judicious OPAT discharges instead. An ASP-led review could evaluate the need for continued antimicrobial therapy, and if oral therapy or de-escalation is an option, could include a review of dosing strategies to promote compliance and convenience. Such a review could ensure that follow-up is arranged when needed. Documenting these items within the ASP workflow can be challenging and requires coordination with case management, IT services, and the discharging teams. Ideally, this review would occur prior to insertion of a peripherally inserted central catheter without delaying hospital discharges.

\section{Antibiotic allergy reconciliation and management}

The overreporting of unconfirmed antibiotic allergies poses considerable harm to patients and a great challenge to antimicrobial stewardship efforts. Patients labeled with a penicillin or $\beta$-lactam allergy are often prescribed suboptimal and excessively broad-spectrum agents, and which have been associated with worse clinical outcomes, increased risk of adverse events, and the promotion of antibiotic resistance. ${ }^{28,29}$ Both frontline pharmacists and antimicrobial stewardship pharmacists are well suited to identify these patients and to determine whether they are eligible for an antibiotic allergy intervention. ${ }^{28}$ This information can be used to risk-stratify patients and inform the appropriate next steps.

Facilities with limited resources may consider interventions requiring minimal personnel time, such as thorough allergy reconciliations or provider education on $\beta$-lactam cross-reactivity rates, potentially coupled with optimizations to the EHR to streamline allergy alerts. Programs with more resources may be able to identify low-risk patients and perform an amoxicillin challenge to remove the allergy from their medical record, ${ }^{30,31}$ while mediumand high-risk groups receive penicillin allergy skin testing (PAST) or desensitization. An abundance of literature supports the successful implementation of PAST, citing numerous approaches that can be adapted to fit institution-specific needs. ${ }^{32}$ 


\section{Pharmacy-driven protocols and collaborative practice agreements}

A collaborative practice agreement (CPA) is a relationship between pharmacists and prescribers to expand the care pharmacists provide independently and can serve several roles in an ASP. Pharmacy-driven protocols also empower greater action by pharmacists. Examples include dosing adjustment, route of administration, and laboratory monitoring for specific medications.

\section{Antimicrobial dosing}

Vancomycin and aminoglycosides require careful monitoring to ensure that the patient receives an adequate dose to treat their infection without toxicity. Beta-lactam antibiotics benefit from prolonged infusion times, which ensure that concentrations remain adequate at the site of infection. Patients with abnormal renal function require regular modifications to antimicrobial dosing. These are all key targets for CPAs that allow pharmacists to adjust medications without provider approval. These pharmacyled dosing strategies save provider time and lead to reduced rates of nephrotoxicity, higher percentage of adequate serum concentrations, cost savings, and improved treatment outcomes. ${ }^{33-37}$

\section{Intravenous to oral conversion of antimicrobial therapy}

Conversion of antimicrobial administration from the intravenous route to the oral (PO) route (IV to $\mathrm{PO}$ ) is one of the simplest expanded practices. Early IV-to-PO conversion leads to decreases in adverse effects, LOS, and cost. ${ }^{38}$ Although it is regarded as one of the more accessible interventions for new ASPs, a procedure outlining settings for appropriate use is crucial in maximizing impact and minimizing the risk to patient safety. When developed carefully, an IV-to-PO intervention can be implemented with minimal resources without requiring ID expertise.

\section{Nursing and antimicrobial stewardship}

Antimicrobial stewardship efforts crosscut all venues of patient care, and interventions utilizing existing workflow hold tremendous potential. Bedside nurses are well positioned to participate in antimicrobial stewardship activities as part of a multidisciplinary approach. However, survey results suggest that although nurses consider antimicrobial stewardship important, knowledge gaps exist. ${ }^{39}$ As awareness grows, potential roles for nurse involvement in antimicrobial stewardship efforts will continue to expand. ${ }^{40}$

Nurses view antimicrobial stewardship as a natural extension of their role as patient advocates. ${ }^{39}$ Nurses are well positioned to question the appropriateness of testing, proper culture technique, antibiotic allergies, and to identify adverse antibiotic events. ${ }^{41}$ Barriers to successful implementation include inadequate education, time constraints, and cultural perceptions about healthcare roles that impede safe multidisciplinary communication.

Infection preventionists are also well suited to make important contributions toward successful antimicrobial stewardship. More training in microbiology and resistance makes the infection preventionist an ideal nurse educator for antimicrobial stewardship and a valuable interface between nursing and other antimicrobial stewardship leaders. Infection preventionists are also aware of local facility epidemiology and testing procedures that can play an important role in developing antimicrobial stewardship interventions tailored to the unique needs at each institution, thereby circumventing negative trends in resistance before they become widespread.

\section{Electronic clinical decision support systems in antimicrobial stewardship}

Electronic CDSSs are computer applications designed to present data at the point of care to guide clinical decisions. Since the first computerized CDSS for antibiotic selection in $1998,{ }^{42}$ multiple studies have shown this technology to improve clinical management of infections. The use of CDSS as an antimicrobial stewardship tool has rapidly expanded with the universal adoption of the EHRs and integration of IT in healthcare. Given the limited resources for antimicrobial stewardship personnel, CDSS is an attractive method to both facilitate compliance and improve efficiency. ${ }^{43}$ However, the sheer volume, variety, and required maintenance of antimicrobial stewardship-related CDSS options can be imposing, and decisions regarding adoption of CDSS tools should be carefully considered.

Standalone CDSS programs with no EHR integration include institutional antibiograms, reports in antimicrobial resistance trends and utilization, and clinical prediction rules and care pathways. ${ }^{44}$ Commercially available programs can pull data from the EHR in real time and can provide facility surveillance and PAF in the form of targeted alerts, as well as medication dosing assistants and data tracking. These programs can expand surveillance and improve intervention efficiency, although they are often hindered by significant cost and limited customizability. ${ }^{45}$ These tools can also be built directly into the EHR, ranging from alerts triggered upon ordering specific agents or flagging medication interaction, to providing reminders of guideline recommendations for prescribing and testing various disease states at the point of ordering. ${ }^{46-48}$ Selecting combinations of these tools based on resources and needs can optimize an institution's ability to leverage technology to bolster the impact of its ASP.

\section{Handshake stewardship}

Handshake stewardship is a unique rounding strategy that consists of in-person rounds during which feedback is provided by a pharmacist-provider team about antibiotic use in each individual patient, without restriction or prior authorization of antibiotics. ${ }^{49}$ This strategy has been shown to decrease anti-infective use without detrimental effects on severity adjusted mortality, readmissions, or lengths of stay while also promoting a collaborative relationship between prescribers and the ASP. ${ }^{50}$

\section{Antimicrobial stewardship in special populations}

Antimicrobial stewardship can be particularly challenging in highrisk populations, including the critically ill and immunocompromised. These patients have higher incidence of multidrug-resistant pathogens due to cumulative healthcare contact and antibiotic exposure, and prescribing practices in these groups tend to be liberal in spectrum and volume. Nevertheless, antimicrobial resistance is now a well-recognized threat to the success of transplant and oncology programs, and ASPs that recognize and adapt to these challenges can be successful in these populations. ${ }^{51}$

A top priority is understanding unique factors that affect ID management in these patients, providing antimicrobial stewardship teams with valuable insights into prescribing behaviors. Armed with this information, antimicrobial stewardship teams may choose a more permissive approach to empiric broadspectrum use and, rather, focus efforts on appropriate dosing, toxicity avoidance, and early de-escalation. In other situations, 
competing considerations such as palliative versus curative care or transplant candidacy may affect antimicrobial choices. ${ }^{51}$

The global coronavirus disease 2019 (COVID-19) pandemic has effectively demonstrated the importance of an established ASP in managing a new cohort of unique patients. Hospitals with an established PAF program can and have used it for collaborative review of complex COVID-19 patients, even as global understanding of appropriate management has unfolded. Discussions regarding de-escalation of antibiotics and approval of novel therapeutics (eg, remdesivir, tocilizumab) can be facilitated within such a systematic approach, increasing efficiency, consistency, and equity in disease management even during a global health crisis.

In our experience, developing relationships with the respective clinical leaders of these unique populations is the single best antimicrobial stewardship strategy for sustained success. One effective method is integrating the antimicrobial stewardship team member into multidisciplinary rounds. This integration facilitates real-time antimicrobial stewardship recommendations that are more collaborative than traditional PAF. Recent research exploring antimicrobial stewardship in immunocompromised cohorts confirms that availability and consistency of recommendations are key to success. ${ }^{53}$ Other activities that develop key relationships include involving ICU and transplant leaders in the institution's antimicrobial stewardship committee, codeveloping care pathways, and collaborating on antimicrobial stewardship-related research. Antimicrobial stewardship participation in creating algorithms and care pathways is an efficient method of influencing prescribing.

Finally, using local data to generate unit or program specific antibiograms to guide appropriate antimicrobial selection is one relatively accessible option in a high-risk population, and resistance trends can help justify systematic changes in prescribing for common conditions where a predominant pathogen is known. ${ }^{54}$

In conclusion, all hospitals can implement practices to improve antimicrobial prescribing, regardless of size, financial support, and resource allocation. Identifying opportunities for clinical improvement of patients will allow ASPs to incrementally improve patient care. Keys to success include setting attainable goals; focusing on evidence-based fundamental antimicrobial stewardship interventions; fostering positive and productive relationships; and adapting expanded practice antimicrobial stewardship interventions to the local environment and resources.

\section{Acknowledgments.}

Financial support. No financial support was provided relevant to this article.

Conflicts of interest. All authors report no conflicts of interest relevant to this article.

\section{References}

1. Checklist for core elements of hospital antibiotic stewardship programs. Centers for Disease Control and Prevention website. https://www.cdc. gov/antibiotic-use/core-elements/hospital/checklist.html. Published 2019. Accessed October 18, 2019.

2. Tamma PD, Miller MA, Cosgrove SE. Rethinking how antibiotics are prescribed: incorporating the 4 moments of antibiotic decision making into clinical practice. JAMA 2019;321:139-140.

3. Szymczak JN, J. The social determinants of antibiotic prescribing: implications for the development and implementation of stewardship interventions. In: Barlam TN, MM; Tamma, PD; Trivedi, KK, ed. Practical
Implementation of an Antibiotic Stewardship Program. Cambridge University Press Online; 2018.

4. Baker DW, Hyun D, Neuhauser MM, Bhatt J, Srinivasan A. Leading practices in antimicrobial stewardship: conference summary. Jt Comm J Qual Patient Saf 2019;45:517-523.

5. Magill SS, Edwards JR, Beldavs ZG, et al. Prevalence of antimicrobial use in US acute care hospitals, May-September 2011. JAMA 2014;312:1438-1446.

6. Glowacki RC, Schwartz DN, Itokazu GS, Wisniewski MF, Kieszkowski P, Weinstein RA. Antibiotic combinations with redundant antimicrobial spectra: clinical epidemiology and pilot intervention of computer-assisted surveillance. Clin Infect Dis 2003;37:59-64.

7. Evans RS, Olson JA, Stenehjem E, et al. Use of computer decision support in an antimicrobial stewardship program (ASP). Appl Clin Inform 2015;6:120135.

8. Bessesen, MT. Antimicrobial stewardship programs comparison of a program with infectious diseases pharmacist support to a program with a geographic pharmacist staffing model. Hosp Pharm 2015;50:477-483.

9. Shaughnessy MK, Amundson WH, Kuskowski MA, DeCarolis DD, Johnson JR, Drekonja DM. Unnecessary antimicrobial use in patients with current or recent Clostridium difficile infection. Infect Control Hosp Epidemiol 2013;34:109-116.

10. Timbrook TT, Morton JB, McConeghy KW, Caffrey AR, Mylonakis E, LaPlante KL. The effect of molecular rapid diagnostic testing on clinical outcomes in bloodstream infections: a systematic review and meta-analysis. Clin Infect Dis 2017;64:15-23.

11. Wenzler E, Timbrook TT, Wong JR, Hurst JM, MacVane SH. Implementation and optimization of molecular rapid diagnostic tests for bloodstream infections. Am J Health Syst Pharm 2018;75:1191-1202.

12. Reed EE, Stevenson KB, West JE, Bauer KA, Goff DA. Impact of formulary restriction with prior authorization by an antimicrobial stewardship program. Virulence 2013;4:158-162.

13. Gross R, Morgan AS, Kinky DE, Weiner M, Gibson GA, Fishman NO. Impact of a hospital-based antimicrobial management program on clinical and economic outcomes. Clin Infect Dis 2001;33:289-295.

14. Seemungal IA, Bruno CJ. Attitudes of housestaff toward a prior-authorization-based antibiotic stewardship program. Infect Control Hosp Epidemiol 2012;33:429-431.

15. Szymczak JE, Kitt E, Hayes M, et al. Threatened efficiency not autonomy: Prescriber perceptions of an established pediatric antimicrobial stewardship program. Infect Control Hosp Epidemiol 2019;40:522-527.

16. Tamma PD, Avdic E, Keenan JF, et al. What is the more effective antibiotic stewardship intervention: preprescription authorization or postprescription review with feedback? Clin Infect Dis 2017;64:537-543.

17. Anderson DJ, Watson S, Moehring RW, et al. Feasibility of core antimicrobial stewardship interventions in community hospitals. JAMA Netw Open 2019;2:e199369.

18. Ball JR, Balogh E. Improving diagnosis in health care: highlights of a report from the national academies of sciences, engineering, and medicine. Ann Intern Med 2016;164:59-61.

19. Madden GR, Weinstein RA, Sifri CD. Diagnostic stewardship for healthcare-associated infections: opportunities and challenges to safely reduce test use. Infect Control Hosp Epidemiol 2018;39:214-218.

20. Avdic E, Cushinotto LA, Hughes AH, et al. Impact of an antimicrobial stewardship intervention on shortening the duration of therapy for community-acquired pneumonia. Clin Infect Dis 2012;54:1581-1587.

21. Jenkins TC, Knepper BC, Moore SJ, et al. Antibiotic prescribing practices in a multicenter cohort of patients hospitalized for acute bacterial skin and skin structure infection. Infect Control Hosp Epidemiol 2014;35:1241-1250.

22. Vaughn VM, Flanders SA, Snyder A, et al. Excess antibiotic treatment duration and adverse events in patients hospitalized with pneumonia: a multihospital cohort study. Ann Intern Med 2019;171:153-163.

23. Feller J, Lund BC, Perencevich EN, et al. Postdischarge oral antimicrobial use among hospitalized patients across an integrated national healthcare network. Clin Microbiol Infect 2020;26:327-332.

24. Vaughn VM, Gandhi TN, Chopra V, et al. Antibiotic overuse after hospital discharge: a multi-hospital cohort study. Clin Infect Dis 2020. doi: 10.1093/ cid/ciaa1372. 
25. Williams DN, Baker CA, Kind AC, Sannes MR. The history and evolution of outpatient parenteral antibiotic therapy (OPAT). Int J Antimicrob Agents 2015;46:307-312.

26. Spivak ES, Kendall B, Orlando P, et al. Evaluation of outpatient parenteral antimicrobial therapy at a Veterans' Affairs hospital. Infect Control Hosp Epidemiol 2015;36:1103-1105.

27. Gordon SM, Shrestha NK, Rehm SJ. Transitioning antimicrobial stewardship beyond the hospital: the Cleveland Clinic's community-based parenteral anti-infective therapy (COPAT) program. J Hosp Med 2011;6 suppl 1:S24-S30.

28. Shenoy ES, Macy E, Rowe T, Blumenthal KG. Evaluation and management of penicillin allergy: a review. JAMA 2019;321:188-199.

29. Blumenthal KG, Peter JG, Trubiano JA, Phillips EJ. Antibiotic allergy. Lancet 2019;393:183-198.

30. Mustafa SS, Conn K, Ramsey A. Comparing direct challenge to penicillin skin testing for the outpatient evaluation of penicillin allergy: a randomized controlled trial. J Allergy Clin Immunol Pract 2019;7:2163-2170.

31. Vyles D, Macy E. Self-reported beta-lactam intolerance: not a class effect, dangerous to patients, and rarely allergy. Expert Rev Anti Infect Ther 2019;17:429-435.

32. Bland CM, Bookstaver PB, Griffith NC, et al. A practical guide for pharmacists to successfully implement penicillin allergy skin testing. Am J Health Syst Pharm 2019;76:136-147.

33. Momattin H, Zogheib M, Homoud A, Al-Tawfiq JA. Safety and outcome of pharmacy-led vancomycin dosing and monitoring. Chemotherapy 2016; 61:3-7.

34. Smith AP, Millares-Sipin CA, James M, Cohen H. Impact of a pharmacistinitiated vancomycin monitoring program. Consult Pharm 2016;31:505-510.

35. Bond CA, Raehl CL. Clinical and economic outcomes of pharmacist-managed aminoglycoside or vancomycin therapy. Am J Health Syst Pharm 2005;62:1596-1605.

36. Falagas ME, Tansarli GS, Ikawa K, Vardakas KZ. Clinical outcomes with extended or continuous versus short-term intravenous infusion of carbapenems and piperacillin/tazobactam: a systematic review and meta-analysis. Clin Infect Dis 2013;56:272-282.

37. Olson J, Hersh AL, Sorensen J, Zobell J, Anderson C, Thorell EA. Intravenous vancomycin therapeutic drug monitoring in children: evaluation of a pharmacy-driven protocol and collaborative practice agreement. J Pediatr Infect Dis Soc 2020;9:334-341.

38. Laing RB, Mackenzie AR, Shaw H, Gould IM, Douglas JG. The effect of intravenous-to-oral switch guidelines on the use of parenteral antimicrobials in medical wards. J Antimicrob Chemother 1998;42:107-111.

39. Merrill K, Hanson SF, Sumner S, Vento T, Veillette J, Webb B. Antimicrobial stewardship: staff nurse knowledge and attitudes. Am J Infect Control 2019;47:1219-1224.
40. Olans RN, Olans RD, DeMaria A Jr. The critical role of the staff nurse in antimicrobial stewardship_-unrecognized, but already there. Clin Infect Dis 2016;62:84-89.

41. Monsees E, Popejoy L, Jackson MA, Lee B, Goldman J. Integrating staff nurses in antibiotic stewardship: opportunities and barriers. Am J Infect Control 2018;46:737-742.

42. Evans RS, Pestotnik SL, Classen DC, et al. A computer-assisted management program for antibiotics and other antiinfective agents. $N$ Engl J Med 1998;338:232-238.

43. Rittmann B, Stevens MP. Clinical decision support systems and their role in antibiotic stewardship: a systematic review. Curr Infect Dis Rep 2019;21:29.

44. Webb BJ, Sorensen J, Mecham I, et al. Antibiotic use and outcomes after implementation of the drug resistance in pneumonia score in ED Patients with community-onset pneumonia. Chest 2019;156:843-851.

45. Kuper KM, Nagel JL, Kile JW, May LS, Lee FM. The role of electronic health record and "add-on" clinical decision support systems to enhance antimicrobial stewardship programs. Infect Control Hosp Epidemiol 2019;40:501-511.

46. Keller SC, Feldman L, Smith J, Pahwa A, Cosgrove SE, Chida N. The use of clinical decision support in reducing diagnosis of and treatment of asymptomatic bacteriuria. J Hosp Med 2018;13:392-395.

47. Mizusawa M, Small BA, Hsu YJ, et al. Prescriber behavior in Clostridioides difficile testing: a 3-hospital diagnostic stewardship intervention. Clin Infect Dis 2019;69:2019-2021.

48. Shekelle PG, Morton SC, Keeler EB. Costs and benefits of health information technology. Evid Rep Technol Assess (Full Rep) 2006;134: $1-71$.

49. Hurst AL, Child J, Pearce K, et al. Handshake stewardship: a highly effective rounding-based antimicrobial optimization service. Pediatr Infect Dis J 2016;35:1104-1110.

50. McBrayne CE, Williams MC, Levek C, et al. Sustainability of handshake stewardship: extending a hand is effective years later. Clin Infect Dis 2020;70:2325-2332.

51. Abbo LM, Ariza-Heredia EJ. Antimicrobial stewardship in immunocompromised hosts. Infect Dis Clin North Am 2014;28:263-279.

52. Taur Y, Xavier JB, Lipuma L, et al. Intestinal domination and the risk of bacteremia in patients undergoing allogeneic hematopoietic stem cell transplantation. Clin Infect Dis 2012;55:905-914.

53. Tverdek FP, Rolston KV, Chemaly RF. Antimicrobial stewardship in patients with cancer. Pharmacotherapy 2012;32:722-734.

54. Ford CD, Coombs J, Stofer MG, et al. Decrease in vancomycin-resistant Enterococcus colonization associated with a reduction in carbapenem use as empiric therapy for febrile neutropenia in patients with acute leukemia. Infect Control Hosp Epidemiol 2019;40:774-779. 